
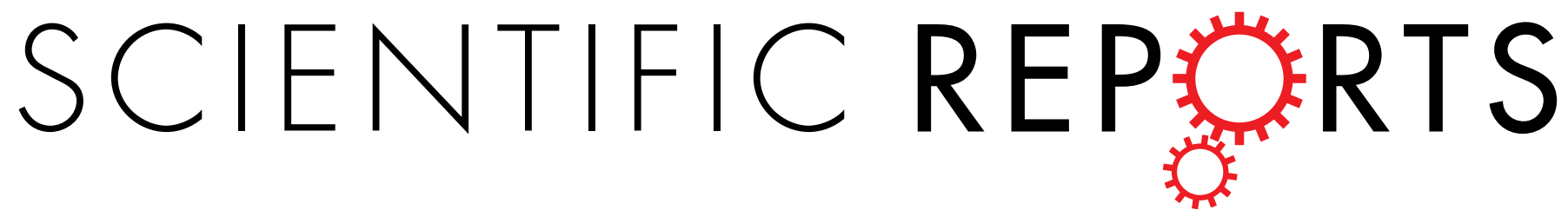

OPEN

\title{
Genetic and Epigenetic Alterations of TERT Are Associated with Inferior Outcome in Adolescent and Young \\ Adult Patients with Melanoma
}

Received: 18 August 2016

Accepted: 03 March 2017

Published: 05 April 2017

\author{
Brittani Seynnaeve ${ }^{1, *}$, Seungjae Lee ${ }^{2, *}$, Sumit Borah ${ }^{2}$, Yongseok Park ${ }^{3}$, Alberto Pappo ${ }^{4}$, \\ John M. Kirkwood ${ }^{5}$ \& Armita Bahrami ${ }^{2,4}$
}

Progression of melanoma to distant sites in adolescents and young adults (AYAs) is not reliably predicted by clinicopathologic criteria. TERT promoter mutations when combined with BRAFINRAS mutations correlate with adverse outcome in adult melanoma. To determine the prognostic value of TERT alterations in AYA melanoma, we investigated the association of TERT promoter mutations, as well as promoter methylation, an epigenetic alteration also linked to TERT upregulation, with TERT mRNA expression and outcome using a well-characterized cohort of 27 patients with melanoma (ages 8-25, mean 20). TERT mRNA expression levels were significantly higher in tumors harboring TERT promoter mutation and/or hypermethylation than those without either aberration $(P=0.046)$. TERT promoter mutations alone did not predict adverse outcomes $(P=0.50)$, but the presence of TERT promoter methylation, alone or concurrent with promoter mutations, correlated with reduced recurrence-free survival $(P=0.001)$. These data suggest that genetic and epigenetic alterations of TERT are associated with TERT upregulation and may predict clinical outcomes in AYA melanoma. A more exhaustive understanding of the different molecular mechanisms leading to increased TERT expression may guide development of prognostic assays to stratify AYA melanoma patients according to clinical risk.

Despite stable or declining incidence rates for most types of cancer in the US, the rate of pediatric and adolescent melanoma has increased from the 1970 s to $2009^{1-5}$. Although recent reports indicate a mitigation of this trend ${ }^{6-8}$, melanoma remains one of the most commonly occurring solid tumors in adolescents and young adults (AYAs) aged 15-29 years $^{9-11}$, accounting for $4 \%$ of all cancers diagnosed in this age group ${ }^{12}$. AYAs with cancer suffer from poorer care and a lag in outcome improvements and it is unclear whether this group should be classified and treated as similarly to older adults, younger pediatric patients, or as a unique subgroup altogether ${ }^{13-15}$.

In contrast to the static situation in AYA, advances in understanding the genomics of adult melanoma have changed the treatment paradigm for advanced staged melanoma in adults. For example, approximately $50 \%$ of adult melanomas carry an oncogenic $B R A F^{\mathrm{V} 600}$ mutation and approximately $20 \%$ carry an oncogenic $N R A S$ mutation ${ }^{16}$, prompting use of selective inhibitors which target the mitogen-activated protein kinase (MAPK) and phosphatidylinositol 3-kinase-AKT pathways ${ }^{17-19}$. Mutations of the TERT promoter, often in combination with $B R A F$ or NRAS mutations, also frequently occur in melanoma ${ }^{20,21}$ and correlate with poorer prognosis, adverse prognostic indicators at the primary site, and lower overall survival ${ }^{22-28}$. Other genetic and epigenetic aberrations of TERT have also been documented in melanoma, such as copy number amplification and promoter hypermethylation ${ }^{29-31}$.

${ }^{1}$ Department of Pediatric Hematology/Oncology, Children's Hospital of Pittsburgh of University of Pittsburgh Medical Center, Pittsburgh, Pennsylvania 15224, USA. ${ }^{2}$ Department of Pathology, St. Jude Children's Research Hospital, Memphis, Tennessee 38105, USA. ${ }^{3}$ Department of Biostatistics, University of Pittsburgh, Pittsburgh, Pennsylvania 15261, USA. " Department of Oncology, St. Jude Children's Research Hospital, Memphis Tennessee 38105, USA. ${ }^{5}$ Department of Hematology/Oncology, University of Pittsburgh Medical Center, Pittsburgh, Pennsylvania 15213, USA. *These authors contributed equally to this work. Correspondence and requests for materials should be addressed to A.B. (email: armita.bahrami@stjude.org) 

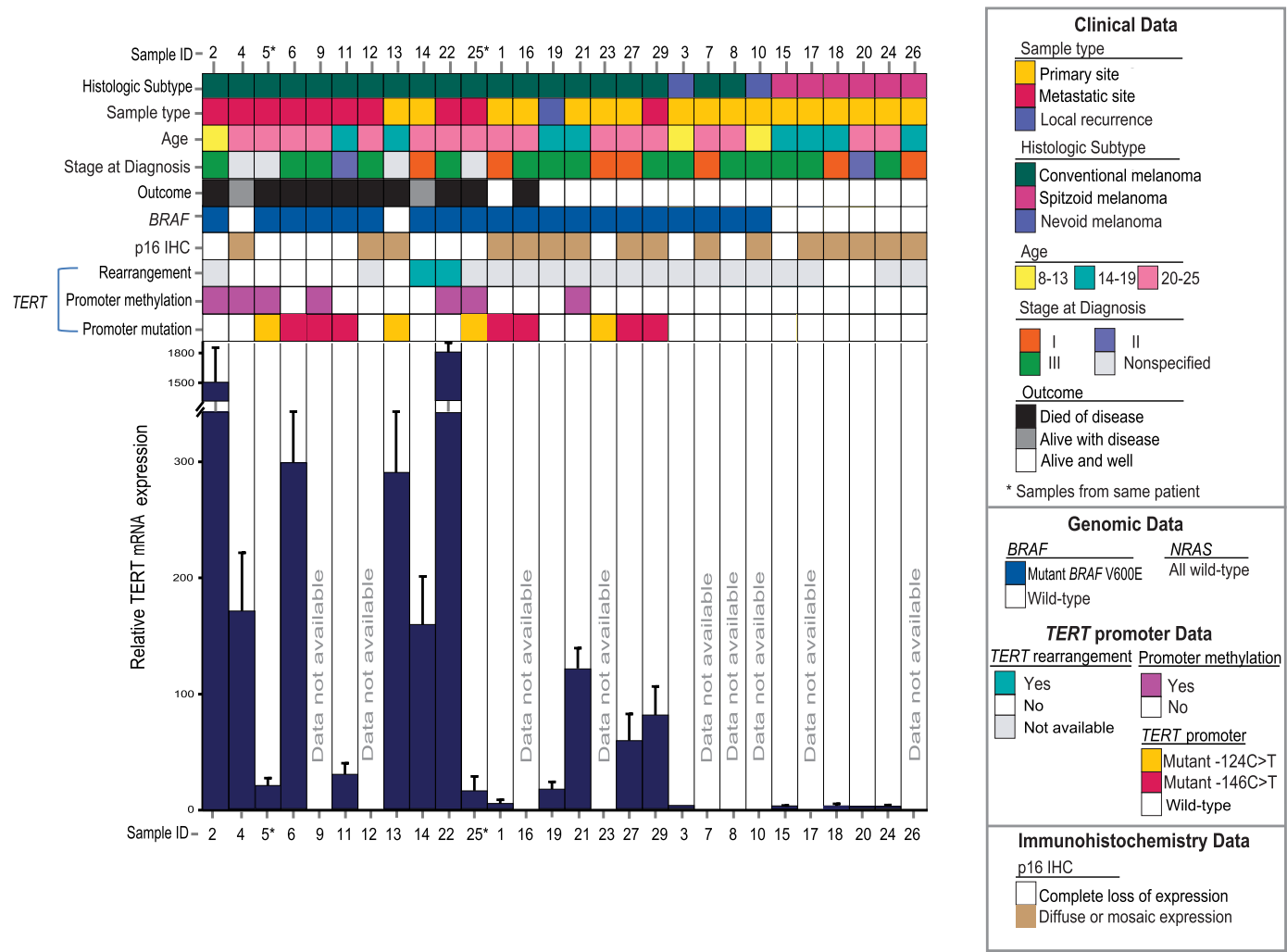

Figure 1. Relative TERT mRNA expression by RT-qPCR and the associated genomic, clinical, and outcome data for 28 melanoma samples from AYA patients.

Unfortunately it is unclear to what extent the data derived from adult melanoma studies are relevant for AYA melanoma since biological differences between age groups may influence tumor characteristics and patient outcome. A recent genomic study of 23 pediatric melanomas revealed that adolescent and adult conventional melanomas are similar in that both (i) have a high burden of ultraviolet-induced signature mutations, (ii) commonly harbor activating mutations in $B R A F$ and the TERT promoter, and (iii) commonly harbor inactivating alterations of the CDKN2A and PTEN tumor suppressor genes ${ }^{32}$. Given the association of TERT promoter mutations with adverse outcome in adult melanoma patients ${ }^{22}$, we investigated the prognostic value of these mutations, as well as promoter hypermethylation - an epigenetic alteration linked to TERT upregulation in a subset of melanomas ${ }^{29}$, using 28 tissue specimens from a well-annotated cohort of 27 AYA melanomas at the University of Pittsburgh. This cohort included cases of conventional melanoma $(n=20)$, nevoid melanoma $(n=2)$, and spitzoid melanoma $(n=6)$. We also measured TERT mRNA expression levels and screened for mutations in BRAF, NRAS and loss of p16 expression. A subset of these samples were additionally screened for genomic rearrangement involving TERT. The prognostic value of these for recurrence-free survival and overall survival was then calculated.

\section{Results}

TERT Promoter Mutations. Sequencing of the TERT promoter revealed that 10 of 19 (53\%) conventional melanomas harbored promoter mutations ( 3 cases of $-124 \mathrm{C}>\mathrm{T}$ and 7 cases of $-146 \mathrm{C}>\mathrm{T}$; Fig. 1 ). Results for the 2 samples from the same conventional melanoma patient were identical. None of the nevoid or spitzoid melanomas contained these mutations. The rs $2853669-245 \mathrm{~A}>\mathrm{G}$ single nucleotide polymorphism was present in 14 of 27 (52\%) of all patients and in 11 of $19(58 \%)$ cases of conventional melanoma (Supplementary Table 1).

TERT Promoter Methylation Analysis. MassARRAY revealed that 8 of 19 (42\%) cases of conventional melanomas and none of the nevoid or spitzoid melanomas harbored hypermethylated CpG dinucleotides in the Upstream of the Transcription Start Site (UTSS) region of the TERT promoter (Supplementary Table 2); hypermethylation in this region has been shown to correlate with increased TERT expression and poorer patient outcome in a number of different cancers ${ }^{33}$. Those samples for which the average methylation of the five UTSS CpG dinucleotides was above $15 \%$ were considered as having hypermethylated TERT promoter, as per Castelo-Branco et $a l .{ }^{33}$. In order to additionally verify that all five of these CpG dinucleotides were methylated in cis on the same TERT promoter in each of these samples, the corresponding UTSS amplicon was cloned and approximately 20 clones from each sample were sequenced. For two of the samples identified as 'hypermethylated' by MassARRAY (ID\#6 and ID\#16), none of the sequenced clones harbored all five CpG dinucleotides methylated (Supplementary Figure 1). These samples were thus considered to not have hypermethylated TERT promoter. 
TERT mRNA Expression. RNA of a sufficiently high quality for reverse transcription and quantitative PCR (RT-qPCR) analysis was extracted from 14 of 19 conventional melanomas ( 8 with mutated TERT promoter; 6 with wild-type TERT promoter), 1 nevoid melanoma, and 4 spitzoid melanomas. The level of TERT mRNA expression was highly variable among the conventional melanomas and low or undetectable in the nevoid and spitzoid melanomas. TERT mRNA levels in the conventional melanomas were 4- to 300-fold (median, 69-fold) above the reference sample (spitzoid melanoma ID\#20), while two of these samples (ID\#2 and \#22) had extremely high expression levels as compared with the other samples. Median relative TERT mRNA level was significantly higher in cases of conventional melanoma than in nevoid or spitzoid samples $\left(P=1.2 \times 10^{-6}\right)$ and in tumors harboring TERT promoter mutation and/or hypermethylation than those without either aberration $(P=0.046)$.

BRAF and NRAS Mutations. Seventeen of the 19 (89\%) conventional melanomas and both nevoid melanomas harbored the activating $B R A F^{\mathrm{V} 600 \mathrm{E}}$ mutation. All 6 spitzoid melanomas had wild-type BRAF. No NRAS mutations were observed. Identical results were obtained for the 2 samples which were obtained from the same patient (Fig. 1).

p16 Immunohistochemistry. p16 expression was assayed by immunohistochemical analysis. 10 of the 19 (53\%) conventional melanomas were scored as negative for expression or as having only rarely-observed positive cells, consistent with biallelic deletions, inactivating mutations, or epigenetic alterations of CDKN2A in these cases. Results were similar for the 2 samples from the same patient. p16 was also undetectable in 1 of $2(50 \%)$ nevoid melanomas and 1 of 6 (17\%) spitzoid melanomas (Fig. 1).

TERT Break-apart Assay. Fluorescence in situ hybridization revealed that 2 of 8 conventional melanomas tested harbored rearrangements involving the TERT locus (Fig. 2). Rearrangements involving TERT were not observed in either of the 2 spitzoid melanomas tested (Fig. 2).

Association Analyses. TERT promoter mutation and/or methylation was significantly more common in conventional melanoma than in spitzoid or nevoid melanoma $(P=0.001)$. The presence of TERT promoter mutation and/or methylation was not associated with higher stage at presentation $(P=1.00)$, ulceration $(P=1.00)$, nodal metastasis $(P=1.00)$, or increased Breslow thickness $(P=0.58)$. TERT mRNA expression levels were significantly higher in tumors harboring TERT promoter mutation and/or methylation than those without either aberration $(P=0.046)$.

Patients with melanoma harboring TERT promoter mutation and/or methylation had a shorter recurrence-free and overall survival than melanoma patients without these alterations, although these differences did not reach statistical significance $(P=0.06$ and $P=0.08$, respectively). When we analyzed the effect of TERT promoter mutations on survival, no correlation was found with recurrence-free or overall survival $(P=0.50$ and $P=0.38$, respectively). In addition, within the subgroup of melanoma patients harboring TERT promoter mutation, no statistically significant difference in disease-free survival was found between carriers and noncarriers of the rs2853669 $-245 \mathrm{~A}>\mathrm{G}$ polymorphism $(P=0.92$; Supplementary Table 1$)$. The presence of TERT promoter methylation, on the other hand, alone or concurrent with promoter mutations, was significantly associated with reduced recurrence-free survival $(P=0.001)$, but not reduced overall survival $(P=0.06)$. Loss of p16 expression was not associated with either recurrence-free or overall survival $(P=0.26$ and $P=0.63$, respectively).

\section{Discussion}

Our study suggests that TERT promoter alterations, specifically CpG dinucleotide methylation, may have prognostic value in AYA melanoma. A major challenge in treating AYA melanoma is the difficulty in recognizing histologically abnormal melanocytic lesions as unequivocally malignant, even when analyzed by the most experienced pathologists. Although overall survival of adults with melanoma correlates with the stage of disease at diagnosis, the utility of stage alone in AYAs is complicated by increasing incidence of positive lymph nodes at diagnosis in the younger population ${ }^{27,34,35}$. For example, Livestro et al. observed the 5 - and 10-year survival rates of pediatric (mean age 17.2 years) and adult (mean age 53.8 years) cohorts to be similar, despite a higher prevalence of lymph node metastases in the pediatric cohort as compared with the tumor thickness-matched adult control group ${ }^{36}$. Likewise, an initial diagnosis of stage III disease in our cohort did not robustly predict unfavorable outcome, and in fact adverse outcomes were seen in a subset of the patients (ID\#11 and ID\#14) who initially presented with stage I or II disease (Fig. 1). Clinicians are thus often reluctant to treat pediatric and AYA melanoma patients in the same manner as adults with similar pathologic findings, further contributing to difficulties in managing these cases.

For these reasons, effective molecular markers could greatly aid in identifying overtly malignant AYA melanomas and improving patient care in this age group. Griewank et al. demonstrated that TERT promoter mutations independently associate with poor prognosis in primary and metastatic melanoma ${ }^{22}$. Populo et al. showed a similar association of TERT promoter mutations with worse outcome for primary adult melanoma ${ }^{28}$. Macerola et al. found TERT promoter mutations to associate with unfavorable prognostic parameters such as increasing thickness, high mitotic rate, lymph node metastasis, and presence of ulceration when BRAF mutations were also present, although survival analysis was not performed and no significant correlations were found between the presence of promoter mutations and regional and distant metastases ${ }^{24}$. Nagore et al. showed that coexistence of TERT promoter and BRAF or NRAS mutations was associated with a 2 -fold reduced rate of disease-free survival and a 5-fold reduced rate of melanoma-specific survival in patients with stage I and II melanoma ${ }^{26}$. TERT promoter hypermethylation is also an effective molecular marker for prognosis in a number of cancer types, and was previously shown to correlate with increased TERT expression in a subset of melanomas ${ }^{29,33,37-39}$.

In this study we found that TERT promoter methylation, alone, or in combination with promoter mutations, was associated with reduced recurrence-free survival. The significance of this finding should be interpreted 

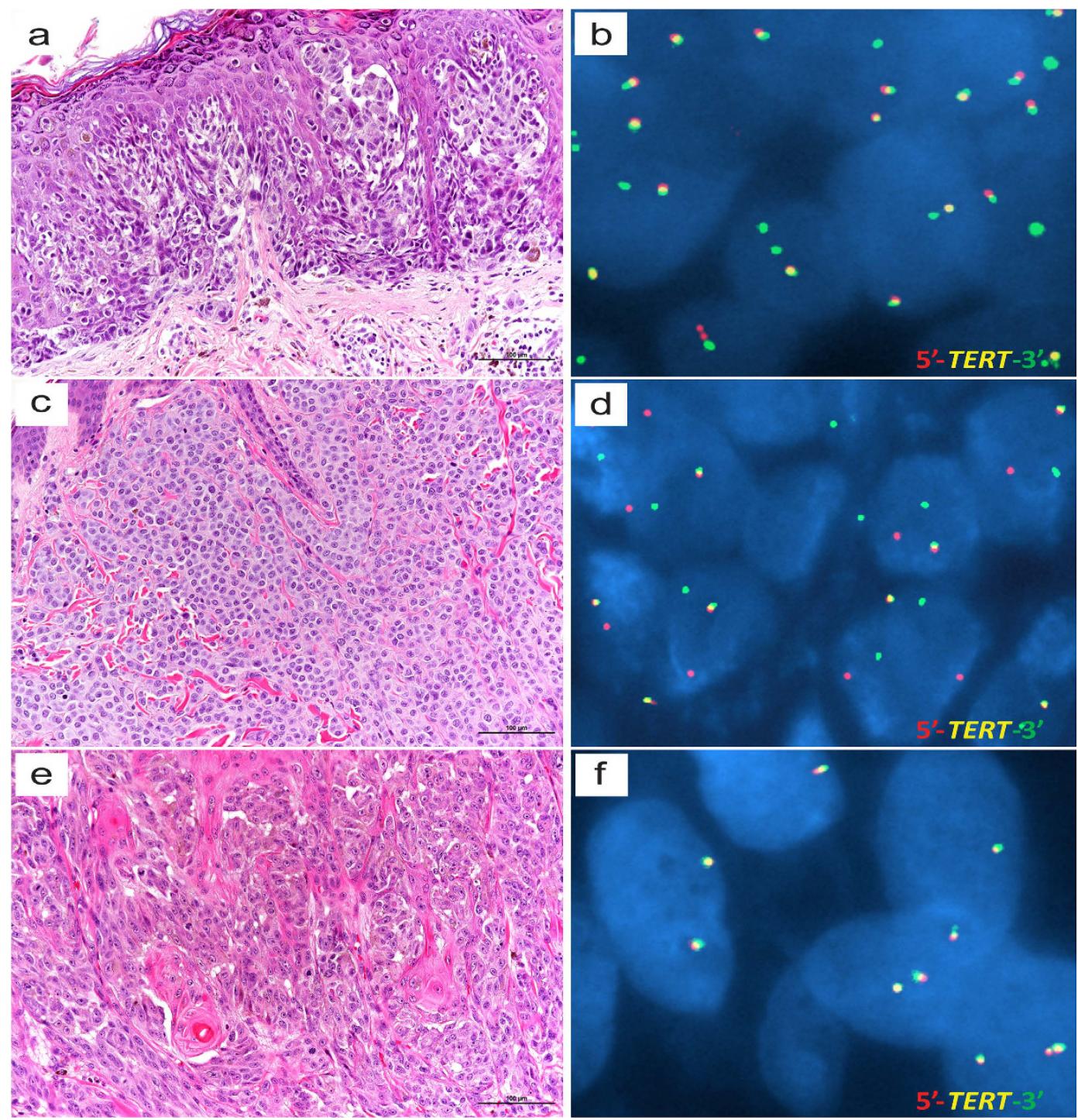

Figure 2. Photomicrographs of hematoxylin and eosin-stained $(H \& E)$ sections and interphase fluorescence in situ hybridization (FISH) with TERT dual color, break-apart probe from AYA patients with melanoma. (a,b) 21-year-old male (ID\#14) with scalp conventional melanoma primary (H\&E, 20×), alive with disease. FISH image (b) suggests TERT rearrangement with deletion of the $5^{\prime}$ TERT in most nuclei. (c,d) 20 -year-old male (ID\#22) with scalp conventional melanoma primary, dermal/subcutaneous metastasis (H\&E, 20×), died of disease. FISH image (d) shows separate red and green signals in most nuclei, consistent with TERT rearrangement. (e,f) 23 -year-old female (ID\#20) with posterior trunk spitzoid melanoma (H\&E, 20×), alive with no evidence of disease. FISH image (f) shows no break-apart (split) signals. Scale bar $=100 \mu \mathrm{m}$.

cautiously, however, due to the heterogeneous samples and small cohort size. In some of these cases, TERT promoter methylation was the sole aberration involving TERT observed, whereas in other cases promoter methylation occurred alongside promoter mutation or rearrangement. For samples harboring both genetic and epigenetic alterations of TERT, the chronological order in which these alterations occurred is not known. Matched primary, recurrent and metastatic melanoma samples would be required for this analysis.

Loss of p16 expression was also not predictive of poor prognosis in this AYA cohort, unlike in adults ${ }^{40,41}$. TERT promoter mutations are acquired before the loss of p16 expression in adult invasive melanoma ${ }^{42}$. Interestingly, however, at least two of these AYA cases had undetectable or low levels of TERT mRNA and complete loss of p16 expression in our study (ID\#3 and ID\#15), suggesting that the order of these events during tumor evolution may be different in a subset of AYA melanomas than in adults. Although the sample size in this study is too small to draw a meaningful conclusion, it is possible that escape from TERT reactivation might occur in a subset of invasive melanoma in AYA patients, and those tumors might follow a biologic course that is different from that in the adult counterpart.

An even higher incidence of BRAF mutation was observed in conventional AYA melanoma patients (89\%) than in adults ${ }^{16}$, in agreement with previous studies, including our own $(87 \%)^{16,32,43,44}$. In a population-based study of 912 cutaneous melanoma patients, higher $(>=\mathrm{T} 2 \mathrm{~b})$ but not lower $(<=\mathrm{T} 2 \mathrm{a})$ stage tumors with either 
NRAS or BRAF mutation had a significantly poorer melanoma-specific survival rate as compared with tumors harboring wild type $N R A S$ and $B R A F$, after adjusting for other prognostic factors. We found no incidence of $N R A S$ mutation in this cohort and did not observe a significant correlation between $B R A F$ mutation and clinical outcome, although our sample size was insufficient for subgroup analysis ${ }^{44}$.

TERT mRNA expression level was significantly higher in cases of conventional melanoma than in nevoid or spitzoid samples. TERT mRNA levels were also significantly higher in melanomas harboring TERT promoter mutation and/or methylation than in those without these alterations. However, technical difficulties in measuring TERT mRNA levels in tumor tissue limit its use as a prognostic marker. RT-qPCR assays require high-quality RNA extracted from highly pure tumor samples, which is often not possible for biopsy samples. Also, although TERT is silenced in the majority of somatic cells, it remains active in some non-cancerous proliferating cells such as stem cells, activated lymphocytes, and hair follicle epithelial cells. Contamination by these cells in biopsy samples can therefore prevent accurate estimation of mRNA levels in actual tumor cells.

In some of the conventional AYA melanoma cases studied here, the TERT promoter was neither mutated nor hypermethylated. Since the frequency of the TERT-independent Alternative Lengthening of Telomere mechanism in melanoma is $l \mathrm{w}^{45}$, and since genomic rearrangement involving TERT is another mechanism for upregulation of this gene ${ }^{46}$, we tested whether a subset of the AYA cases harbored such rearrangements, including those cases which lacked mutated or hypermethylated TERT promoter. Indeed, two instances of TERT rearrangement were observed (Fig. 2); in one of these cases neither mutated nor hypermethylated TERT promoter was detected. Thus, understanding which of the possible genetic or epigenetic pathways for TERT upregulation correlates with especially high levels of TERT expression in melanoma may facilitate development of more reliable prognostic assays. Of note, a relatively high level of TERT mRNA was measured in a primary melanoma which later developed distant metastasis, despite the fact that the tumor harbored a wild-type TERT promoter and was initially diagnosed as stage I disease (ID \# 14; Fig. 1 and 2). This case highlights the danger of using only one of the multiple known DNA-level mechanisms for TERT dysregulation as a proxy for TERT mRNA expression level.

In summary, our study suggests that TERT is upregulated through various genetic and epigenetic alterations in AYA melanoma. The combination of TERT promoter mutation, hypermethylation and, perhaps, rearrangement, effectively serve as a diagnostic proxy for TERT mRNA levels; these DNA-level aberrations are significantly easier to detect in patient samples than mRNA levels. There are a number of limitations to our study. A relatively limited number of samples from a single institution were used in this study. In addition, the follow-up time on several patients was relatively short and the results may not reflect longer term outcome. Although a larger cohort is necessary to confirm these findings, they nevertheless highlight the need for a comprehensive understanding of the of TERT dysregulation in AYA melanoma to develop prognostic assays for clinical risk stratification.

\section{Materials and Methods}

Institutional review board (IRB) approval was obtained from the University of Pittsburgh for patient enrollment in the tissue banking and analysis protocols (UPCI 96-099 and UPCI 15-202) and informed consent was obtained from all subjects. IRB approval was obtained from St. Jude Children's Research Hospital, and studies were conducted in accordance with approved guidelines.

Study Population. Conventional melanomas were identified in 19 patients aged 13-25 years (median, 21.0). There were 10 males and 9 females, of whom 18 were Caucasian and 1 was Indian Asian. Primary tumors arose in the skin of the trunk $(n=8)$, lower extremities $(n=5)$, scalp $(n=4)$, and unspecified site $(n=2)$. Histologic subtypes were as follows: superficial spreading $(n=8)$, nodular $(n=4)$, and not otherwise specified $(n=7)$. At last follow-up (mean, 82.9 months; range, 12-293 months), 9 patients had died of disease, 2 were alive with disease, and 8 were alive with no evidence of disease (Table 1; Fig. 1).

Nevoid melanomas occurred in 2 Caucasian males aged 8 and 13 years. The melanomas arose in the skin of the trunk $(n=2)$. Both patients were alive with no evidence of disease at last follow-up (109 months and 110 months, respectively) (Table 1; Fig. 1).

Spitzoid melanomas occurred in 6 Caucasian females aged 16-23 years (mean, 19.3; median, 18.5; standard deviation, 3.0). The melanomas arose in skin of the lower extremities $(n=3)$, upper extremities $(n=1)$, trunk $(n=1)$, and ear $(n=1)$. All patients were alive with no evidence of disease at last follow-up (mean, 49.0 months; range, 7-96 months) (Table 1; Fig. 1).

Disease Characteristics. The stages of disease (American Joint Committee on Cancer staging system, $7^{\text {th }}$ edition) at diagnosis for patients with conventional melanoma were as follows: stage I $(n=5)$, stage II $(n=1)$, stage III $(n=10)$, and unspecified $(n=3)$. Of the patients with conventional melanoma who underwent sentinel lymph node evaluation at diagnosis $(n=16), 10$ had at least 1 positive node and 6 had no nodal involvement. Of the 19 patients with conventional melanoma, 12 (63\%) had an unfavorable clinical course: distant metastasis $(n=10)$, local recurrence $(n=4)$, and dead of disease in $(n=9)$ (Table 1 and Fig. 1$)$.

The 2 patients with nevoid melanoma were initially diagnosed with the stage III disease, and neither experienced local recurrence or distant metastasis. The stages of disease at diagnosis for the 6 patients with spitzoid melanoma were as follows: stage I $(n=2)$, stage II $(n=1)$, and stage III $(n=3)$. No patients with spitzoid melanoma developed distant metastasis or local recurrence (Fig. 1). Table 1 summarizes the clinical and histopathologic features of patients.

Tissue Specimens. The cohort comprised 27 AYA patients (mean age 20 years) with melanoma who were diagnosed between 1988 and 2014. Tissue specimens were obtained from the pathology archives of the University of Pittsburgh. Inclusion criteria were as follows: (1) lesions showing histologic features of invasive melanoma; (2) 


\begin{tabular}{|c|c|c|c|c|c|c|c|c|c|c|c|c|c|}
\hline $\begin{array}{l}\text { Melanoma } \\
\text { Subtype }\end{array}$ & $\begin{array}{l}\text { Sample } \\
\text { ID }\end{array}$ & Age $^{*}$ & Race & Sex & Primary site & Sample site & $\begin{array}{c}\text { Breslow } \\
(\mathrm{mm})\end{array}$ & Ulcer & $\begin{array}{c}\text { Sentinel LN } \\
\text { metastasis }\end{array}$ & Stage ${ }^{*}$ & $\begin{array}{l}\text { Local } \\
\text { recurrence }\end{array}$ & $\begin{array}{c}\text { Distant } \\
\text { metastasis }\end{array}$ & $\begin{array}{l}\text { Follow-up }{ }^{* * *} \\
\text { (months) }\end{array}$ \\
\hline \multirow{19}{*}{$\begin{array}{l}\text { Conventional } \\
\text { Melanoma }\end{array}$} & 1 & 21 & $\mathrm{C}$ & $\mathrm{F}$ & Left shoulder & Primary & 0.57 & no & no & I & no & no & 56 \\
\hline & 2 & 13 & $\mathrm{C}$ & $\mathrm{M}$ & Scalp & Scalp recurrence & 1.7 & no & yes & III & Yes & yes & 75 \\
\hline & 4 & 25 & IA & $\mathrm{M}$ & Right leg & Soft tissue & NS & NS & NS & NS & Yes & no & 65 \\
\hline & $5 / 25$ & 25 & $\mathrm{C}$ & $\mathrm{F}$ & Left knee & $\begin{array}{c}\text { Brain/soft tissue } \\
\text { arm }\end{array}$ & NS & NS & NS & NS & yes & yes & 205 \\
\hline & 6 & 22 & $\mathrm{C}$ & $\mathrm{M}$ & Scalp & Neck LN & 12 & yes & yes & III & no & yes & 12 \\
\hline & 7 & 21 & $\mathrm{C}$ & $\mathrm{F}$ & Abdomen & Primary & 1.2 & yes & no & I & no & no & 17 \\
\hline & 8 & 24 & $\mathrm{C}$ & $\mathrm{F}$ & Posterior trunk & Primary & 1.1 & no & yes & III & no & no & 50 \\
\hline & 9 & 25 & $\mathrm{C}$ & $\mathrm{F}$ & NS & Soft tissue & NS & NS & yes & III & NS & yes & 52 \\
\hline & 11 & 16 & $\mathrm{C}$ & $\mathrm{F}$ & Right scapula & Soft tissue & 1.9 & yes & no & II & no & yes & 293 \\
\hline & 12 & 24 & C & $\mathrm{F}$ & NS & Lung & 9 & NS & yes & III & NS & yes & 199 \\
\hline & 13 & 18 & $\mathrm{C}$ & $\mathrm{M}$ & Right leg & Primary & 5.35 & yes & NS & NS & no & yes & 24 \\
\hline & 14 & 21 & $\mathrm{C}$ & $\mathrm{M}$ & Scalp & Primary & 1.2 & yes & no & I & no & yes & 67 \\
\hline & 16 & 22 & $\mathrm{C}$ & $\mathrm{M}$ & Anterior trunk & Primary & 3 & yes & yes & III & no & yes & 50 \\
\hline & 19 & 19 & $\mathrm{C}$ & $\mathrm{F}$ & Left thigh & $\begin{array}{l}\text { Soft tissue left } \\
\text { thigh }\end{array}$ & 0.78 & no & yes & III & yes & no & 135 \\
\hline & 21 & 16 & $\mathrm{C}$ & $\mathrm{M}$ & Posterior trunk & Primary & 1.82 & no & yes & III & no & no & 49 \\
\hline & 22 & 20 & $\mathrm{C}$ & $\mathrm{F}$ & Scalp & Soft tissue & NS & NS & yes & III & no & yes & 44 \\
\hline & 23 & 25 & $\mathrm{C}$ & $\mathrm{M}$ & Posterior trunk & Primary & 1 & no & no & I & no & no & 33 \\
\hline & 27 & 25 & $\mathrm{C}$ & $\mathrm{M}$ & Left calf & Primary & 1.65 & no & no & $\mathrm{I}$ & no & no & 96 \\
\hline & 29 & 19 & $\mathrm{C}$ & $\mathrm{M}$ & Anterior trunk & Sentinel LN & 0.9 & no & yes & III & no & no & 54 \\
\hline \multirow{2}{*}{$\begin{array}{l}\text { Nevoid } \\
\text { Melanoma }\end{array}$} & 3 & 8 & $\mathrm{C}$ & $\mathrm{M}$ & Posterior trunk & Primary & 3 & yes & yes & III & no & no & 109 \\
\hline & 10 & 13 & $\mathrm{C}$ & $\mathrm{M}$ & Posterior trunk & Primary & 2.4 & no & yes & III & no & no & 110 \\
\hline \multirow{6}{*}{$\begin{array}{l}\text { Spitzoid } \\
\text { Melanoma }\end{array}$} & 15 & 16 & $\mathrm{C}$ & $\mathrm{F}$ & Right foot & Primary & 5.2 & no & yes & III & no & no & 87 \\
\hline & 17 & 19 & $\mathrm{C}$ & $\mathrm{F}$ & Right ear & Primary & 2.4 & no & yes & III & no & no & 86 \\
\hline & 18 & 17 & $\mathrm{C}$ & $\mathrm{F}$ & Left leg & Primary & 1.35 & no & no & $\mathrm{I}$ & no & no & 8 \\
\hline & 20 & 23 & $\mathrm{C}$ & $\mathrm{F}$ & Posterior trunk & Primary & 2.9 & yes & no & II & no & no & 7 \\
\hline & 24 & 23 & $\mathrm{C}$ & $\mathrm{F}$ & Left hip & Primary & 0.59 & no & yes & III & no & no & 96 \\
\hline & 26 & 18 & $\mathrm{C}$ & $\mathrm{F}$ & Right arm & Primary & 1.32 & no & yes & I & no & no & 10 \\
\hline
\end{tabular}

Table 1. Clinical and histopathologic features for 27 AYA melanoma patients. Abbreviations: C, Caucasian; IA, Indian Asian; NS, not specified; LN, lymph node; *at initial diagnosis; ${ }^{* *}$ follow-up time from date of diagnosis to death or last contact.

patients aged $\leq 25$ years at the time of diagnosis; (3) availability of sufficient tissue for genomic assays; and (4) availability of patient demographic and follow-up information. The study material included 28 formalin-fixed paraffin-embedded (FFPE) tissue specimens: primary tumors $(n=17)$, lymph node metastatic tumors $(n=2)$, and distant metastatic tissues ( $n=9$, including 1 brain, 1 lung, and 7 skin/soft tissue metastatic tumors).

Mutational Analysis of BRAF, NRAS, and TERT Promoter. FFPE tumor sections were manually dissected, with hematoxylin and eosin $(\mathrm{H} \& \mathrm{E})$-stained sections used to guide dissections, to obtain a minimum of $50 \%$ tumor purity in the material before DNA and RNA extraction, as previously described ${ }^{32}$. Mutational hotspots for BRAF (exon 15), NRAS (exons 1 and 2), and a portion of the TERT promoter (HG 19 coordinates, chr5: 1295151-1295347) were screened in genomic DNA of the 28 tumors, as previously described ${ }^{32}$. An additional primer set, using forward primer $5^{\prime}$-CCCACGTGCGCAGCAGGAC-3' and reverse primer $5^{\prime}$-CTCC CAGTGGATTCGCGGGC-3', was also used to detect rare mutations in the TERT promoter (HG 19 coordinates, chr5: 1295131-1295390) ${ }^{23}$. Results were screened by using CLC Main Workbench sequence analysis software version 6.0.2 (CLC bio, Cambridge, MA).

TERT Methylation Analysis. $500 \mathrm{ng}$ of genomic DNA, isolated from FFPE tissue with the Maxwell ${ }^{\circledR} 16$ FFPE Plus LEV DNA Purification Kit (Promega), was processed with the EZ DNA Methylation-Gold ${ }^{\mathrm{TM}} \mathrm{Kit}^{\mathrm{N}}$ (ZYMO RESEARCH), according to the manufacturer's protocol. Sodium bisulfite-treated DNA was used to amplify the TERT promoter, and the degree of methylation was measured by MassARRAY (Agena Bioscience) according to the method of Castelo-Branco et al. Data was analyzed with EpiTYPER. Commercially prepared High Methylated ( $>85 \%$ methylation) and Low Methylated ( $<5 \%$ methylation) Human Genomic DNA (EpigenDx, Hopkinton, MA), and reactions containing no DNA template, were used as controls.

The same PCR product obtained from sodium bisulfite-treated DNA of those samples identified as having hypermethylated UTSS by MassARRAY, as well as one sample identified as having an unmethylated UTSS, were further purified using NucleoSpin Gel and PCR Clean-up Kit (Macherey-Nagel), as per manufacturer's instructions except that the PCR product was mixed with a 2 x volume of $10 \%$ NTI buffer instead of a 2 x volume of $100 \%$ NTI buffer. The product was eluted with $10 \mu \mathrm{L}$ of buffer NE, of which $1 \mu \mathrm{L}$ was incubated with $5 \mu \mathrm{L}$ of $2 x$ GoTaq 
Long PCR Master Mix (Promega) at $95^{\circ} \mathrm{C}$ for 5 minutes and then at $72^{\circ} \mathrm{C}$ for 15 minutes. Three microliters of this volume was used for cloning as per manufacturer's instructions for the TOPO-TA 2.1 cloning system (Invitrogen). Positive clones were identified by blue/white selection and 24 clones from each sample were sequenced.

TERT Break-apart Assay. Chromosomal rearrangements involving the TERT promoter were visualized by fluorescence in situ hybridization using BAC clones CH17-75N21 and CH17-410B01 (BACPAC Resources), as previously described ${ }^{27}$.

TERT Expression by Real-time Quantitative Reverse-Transcription PCR. Total RNA was isolated from FFPE tissue sections by using the Maxwell ${ }^{\circledR} 16$ LEV RNA FFPE Purification Kit (Promega, Madison, WI) and converted to cDNA by using the SuperScript ${ }^{\circledR}$ VILO cDNA Synthesis Kit (Invitrogen, Waltham, MA) according to the manufacturer's protocol. To quantify TERT mRNA expression levels, real-time quantitative reverse-transcription PCR (RT-qPCR) was performed in triplicate using the TaqMan Gene Expression Assay (Life Technologies, Waltham, MA) with primers for TERT (Hs00972656_m1) and GAPDH (Hs02758991_g1) as the endogenous control, using the LightCycler ${ }^{\circledR} 480$ System (Roche, Indianapolis, IN) as previously described ${ }^{47}$. TERT expression was normalized by using a comparative control method of threshold cycles relative to GAPDH expression.

Immunohistochemical Analysis of p16. Representative FFPE tumor blocks of 28 samples were cut in $4-\mu \mathrm{m}$ sections and processed for immunohistochemical analysis, using an antibody directed against p16 (JC8; Santa Cruz Biotechnology, Dallas, TX), as previously described ${ }^{32}$. The immunohistochemical staining of p16 was recorded as follows: i) complete loss of p16 nuclear expression (rare or no positive tumor cells); ii) retained or partial loss of p16 nuclear expression (diffuse or heterogeneous/mosaic staining).

Statistical Analysis. Association analyses between TERT promoter alterations and stage at presentation, melanoma type, ulceration, and nodal metastasis were performed using Fisher exact test. Association analysis between TERT promoter alterations and Breslow thickness was performed using t-test. The influence of immunohistochemical loss of p16 expression, TERT promoter mutations and TERT promoter methylation on patients' survival from diagnosis of melanoma were investigated for the 27 patients. The patient who contributed 2 samples (ID\#5 and ID\#25) was included as only 1 subject for statistical analyses, given very similar genomic data results from the 2 samples. The survival endpoints were overall survival from diagnosis of primary melanoma to death and recurrence-free survival from diagnosis of primary melanoma to the first clinical recurrence of melanoma after definitive treatment. Cases in which the endpoint was not reached at the time of last follow-up were censored at that point. The log-rank test was applied to calculate $p$-values. Statistical analyses were performed using the survival package within R statistical analysis software (version 3.2.2).

\section{References}

1. Austin, M. T., Xing, Y., Hayes-Jordan, A. A., Lally, K. P. \& Cormier, J. N. Melanoma incidence rises for children and adolescents: an epidemiologic review of pediatric melanoma in the United States. J Pediatr Surg 48, 2207-2213, doi: 10.1016/j.jpedsurg.2013.06.002 (2013).

2. Hamre, M. R., Chuba, P., Bakhshi, S., Thomas, R. \& Severson, R. K. Cutaneous melanoma in childhood and adolescence. Pediatr Hematol Oncol 19, 309-317, doi: 10.1080/08880010290057327 (2002).

3. Strouse, J. J., Fears, T. R., Tucker, M. A. \& Wayne, A. S. Pediatric melanoma: risk factor and survival analysis of the surveillance, epidemiology and end results database. J Clin Oncol 23, 4735-4741, doi: 10.1200/JCO.2005.02.899 (2005).

4. Wong, J. R., Harris, J. K., Rodriguez-Galindo, C. \& Johnson, K. J. Incidence of childhood and adolescent melanoma in the United States: 1973-2009. Pediatrics 131, 846-854, doi: 10.1542/peds.2012-2520 (2013).

5. Linabery, A. M. \& Ross, J. A. Trends in childhood cancer incidence in the U.S. (1992-2004). Cancer 112, 416-432, doi: 10.1002/ cncr.23169 (2008).

6. Siegel, D. A. et al. Cancer incidence rates and trends among children and adolescents in the United States, 2001-2009. Pediatrics 134, e945-955, doi: 10.1542/peds.2013-3926 (2014).

7. Campbell, L. B. et al. Melanoma Incidence in Children and Adolescents: Decreasing Trends in the United States. J Pediatr 166, 1505-1513, doi: 10.1016/j.jpeds.2015.02.050 (2015).

8. Barr, R. D. et al. Incidence and incidence trends of the most frequent cancers in adolescent and young adult Americans, including "nonmalignant/noninvasive" tumors. Cancer 122, 1000-1008, doi: 10.1002/cncr.29867 (2016).

9. Children's Oncology Group. \& SEER Program (National Cancer Institute (U.S.)). Cancer epidemiology in older adolescents and young adults 15 to 29 years of age: including SEER incidence and survival, 1975-2000. x, 205 p. <Table of contents only. http://www. loc.gov/catdir/toc/fy0612/2006415458.html (2006).

10. Bleyer, A., Viny, A. \& Barr, R. Cancer in 15- to 29-year-olds by primary site. Oncologist 11, 590-601, doi: 10.1634/ theoncologist.11-6-590 (2006).

11. Averbook, B. J. et al. Pediatric melanoma: analysis of an international registry. Cancer 119, 4012-4019, doi: 10.1002/cncr.28289 (2013).

12. Siegel, R. L., Miller, K. D. \& Jemal, A. Cancer statistics, 2016. CA Cancer J Clin 66, 7-30, doi: 10.3322/caac.21332 (2016).

13. Bleyer, A., Ulrich, C. \& Martin, S. Young adults, cancer, health insurance, socioeconomic status, and the Patient Protection and Affordable Care Act. Cancer 118, 6018-6021, doi: 10.1002/cncr.27685 (2012).

14. Sender, L. \& Zabokrtsky, K. B. Adolescent and young adult patients with cancer: a milieu of unique features. Nat Rev Clin Oncol 12, 465-480, doi: 10.1038/nrclinonc.2015.92 (2015).

15. Bleyer, W. A. Potential favorable impact of the affordable care act of 2010 on cancer in young adults in the United States. Cancer J 16, 563-571, doi: 10.1097/PPO.0b013e3181ff6509 (2010).

16. Cancer Genome Atlas, N. Genomic Classification of Cutaneous Melanoma. Cell 161, 1681-1696, doi: 10.1016/j.cell.2015.05.044 (2015).

17. Davies, H. et al. Mutations of the BRAF gene in human cancer. Nature 417, 949-954, doi: 10.1038/nature00766 (2002).

18. Flaherty, K. T., Yasothan, U. \& Kirkpatrick, P. Vemurafenib. Nat Rev Drug Discov 10, 811-812, doi: 10.1038/nrd3579 (2011)

19. Lee, J. H., Choi, J. W. \& Kim, Y. S. Frequencies of BRAF and NRAS mutations are different in histological types and sites of origin of cutaneous melanoma: a meta-analysis. Br. J. Dermatol. 164, 776-784, doi: 10.1111/j.1365-2133.2010.10185.x (2011). 
20. Horn, S. et al. TERT promoter mutations in familial and sporadic melanoma. Science 339, 959-961 (2013).

21. Huang, F. W. et al. Highly recurrent TERT promoter mutations in human melanoma. Science 339, 957-959, doi: 10.1126/ science.1229259 (2013).

22. Griewank, K. G. et al. TERT promoter mutation status as an independent prognostic factor in cutaneous melanoma. J Natl Cancer Inst 106, doi: 10.1093/jnci/dju246 (2014).

23. Heidenreich, B. et al. Telomerase reverse transcriptase promoter mutations in primary cutaneous melanoma. Nat Commun $\mathbf{5}, 3401$, doi: 10.1038/ncomms4401 (2014).

24. Macerola, E. et al. Coexistence of TERT promoter and BRAF mutations in cutaneous melanoma is associated with more clinicopathological features of aggressiveness. Virchows Arch 467, 177-184, doi: 10.1007/s00428-015-1784-x (2015).

25. Nagore, E. et al. TERT promoter mutations associate with fast growing melanoma. Pigment Cell Melanoma Res, doi: 10.1111/ pcmr.12441 (2015).

26. Nagore, E. et al. TERT promoter mutations in melanoma survival. Int J Cancer 139, 75-84, doi: 10.1002/ijc.30042 (2016).

27. Lee, S. et al. TERT Promoter Mutations Are Predictive of Aggressive Clinical Behavior in Patients with Spitzoid Melanocytic Neoplasms. Sci Rep 5, 11200, doi: 10.1038/srep11200 (2015).

28. Populo, H. et al. TERT promoter mutations in skin cancer: the effects of sun exposure and X-irradiation. J. Invest. Dermatol. 134, 2251-2257, doi: 10.1038/jid.2014.163 (2014).

29. Fan, Y. et al. Telomerase Expression by Aberrant Methylation of the TERT Promoter in Melanoma Arising in Giant Congenital Nevi. J Invest Dermatol 136, 339-342, doi: 10.1038/JID.2015.374 (2016).

30. Diaz, A. et al. TERT gene amplification is associated with poor outcome in acral lentiginous melanoma. J Am Acad Dermatol 71, 839-841, doi: 10.1016/j.jaad.2014.05.035 (2014).

31. Diaz, A. et al. TERT and AURKA gene copy number gains enhance the detection of acral lentiginous melanomas by fluorescence in situ hybridization. J Mol Diagn 16, 198-206, doi: 10.1016/j.jmoldx.2013.10.009 (2014).

32. Lu, C. et al. The genomic landscape of childhood and adolescent melanoma. J Invest Dermatol 135, 816-823, doi: 10.1038/ jid.2014.425 (2015).

33. Castelo-Branco, P. et al. Methylation of the TERT promoter and risk stratification of childhood brain tumours: an integrative genomic and molecular study. Lancet Oncol 14, 534-542, doi: 10.1016/S1470-2045(13)70110-4 (2013).

34. Berk, D. R., LaBuz, E., Dadras, S. S., Johnson, D. L. \& Swetter, S. M. Melanoma and melanocytic tumors of uncertain malignant potential in children, adolescents and young adults-the Stanford experience 1995-2008. Pediatr Dermatol 27, 244-254, doi: 10.1111/j.1525-1470.2009.01078.x (2010)

35. Lallas, A. et al. Atypical Spitz tumours and sentinel lymph node biopsy: a systematic review. Lancet Oncol 15, e178-183, doi: 10.1016/S1470-2045(13)70608-9 (2014).

36. Livestro, D. P. et al. Melanoma in the young: differences and similarities with adult melanoma: a case-matched controlled analysis. Cancer 110, 614-624, doi: 10.1002/cncr.22818 (2007).

37. Bougel, S. et al. Methylation of the hTERT promoter: a novel cancer biomarker for leptomeningeal metastasis detection in cerebrospinal fluids. Clin Cancer Res 19, 2216-2223, doi: 10.1158/1078-0432.CCR-12-1246 (2013).

38. Castelo-Branco, P. et al. A cancer specific hypermethylation signature of the TERT promoter predicts biochemical relapse in prostate cancer: A retrospective cohort study. Oncotarget, doi: 10.18632/oncotarget.10639 (2016).

39. Lindsey, J. C. et al. TERT promoter mutation and aberrant hypermethylation are associated with elevated expression in medulloblastoma and characterise the majority of non-infant SHH subgroup tumours. Acta Neuropathol 127, 307-309, doi: 10.1007/s00401-013-1225-3 (2014).

40. Straume, O., Sviland, L. \& Akslen, L. A. Loss of nuclear p16 protein expression correlates with increased tumor cell proliferation (Ki-67) and poor prognosis in patients with vertical growth phase melanoma. Clin. Cancer Res. 6, 1845-1853 (2000).

41. Lade-Keller, J. et al. Immunohistochemical analysis of molecular drivers in melanoma identifies p16 as an independent prognostic biomarker. J. Clin. Pathol. 67, 520-528, doi: 10.1136/jclinpath-2013-202127 (2014).

42. Shain, A. H. et al. The Genetic Evolution of Melanoma from Precursor Lesions. N Engl J Med 373, 1926-1936, doi: 10.1056/ NEJMoa1502583 (2015).

43. Menzies, A. M. et al. Distinguishing clinicopathologic features of patients with V600E and V600K BRAF-mutant metastatic melanoma. Clin Cancer Res 18, 3242-3249, doi: 10.1158/1078-0432.CCR-12-0052 (2012).

44. Thomas, N. E. et al. Association Between NRAS and BRAF Mutational Status and Melanoma-Specific Survival Among Patients With Higher-Risk Primary Melanoma. JAMA Oncol 1, 359-368, doi: 10.1001/jamaoncol.2015.0493 (2015).

45. Heaphy, C. M. et al. Prevalence of the alternative lengthening of telomeres telomere maintenance mechanism in human cancer subtypes. Am J Pathol 179, 1608-1615, doi: 10.1016/j.ajpath.2011.06.018 (2011).

46. Peifer, M. et al. Telomerase activation by genomic rearrangements in high-risk neuroblastoma. Nature 526, 700-704, doi: 10.1038/ nature14980 (2015)

47. Lee, S., Opresko, P., Pappo, A., Kirkwood, J. M. \& Bahrami, A. Association of TERT promoter mutations with telomerase expression in melanoma. Pigment Cell Melanoma Res, doi: 10.1111/pcmr.12471 (2016)

\section{Acknowledgements}

This research was supported by the National Institutes of Health (National Cancer Institute P30CA021765 and 5T32CA175294-02, and by ALSAC.

\section{Author Contributions}

Conceptualization: B.S., S.B., J.M.K., A.B. Collection and assembly of data: B.S., S.L. Data analysis and interpretations: S.L., Y.P. Writing original draft: B.S., S.B., A.B. Writing-review and editing: S.L., Y.P., S.B., A.P., J.M.K., A.B. Supervision: J.M.K., A.B.

\section{Additional Information}

Supplementary information accompanies this paper at http://www.nature.com/srep

Competing Interests: The authors declare and competing financial interests.

How to cite this article: Seynnaeve, B. et al. Genetic and Epigenetic Alterations of TERT Are Associated with Inferior Outcome in Adolescent and Young Adult Patients with Melanoma. Sci. Rep. 7, 45704; doi: 10.1038/ srep45704 (2017).

Publisher's note: Springer Nature remains neutral with regard to jurisdictional claims in published maps and institutional affiliations. 
(c) (i) This work is licensed under a Creative Commons Attribution 4.0 International License. The images or other third party material in this article are included in the article's Creative Commons license, unless indicated otherwise in the credit line; if the material is not included under the Creative Commons license, users will need to obtain permission from the license holder to reproduce the material. To view a copy of this license, visit http://creativecommons.org/licenses/by/4.0/

(C) The Author(s) 2017 